\title{
Solving Challenging Crystallographic Problems with Automated Electron Diffraction Tomography (ADT)
}

\author{
Ute Kolb $^{1}$, Bastian Barton ${ }^{1}$, Yasar Krysiak ${ }^{1}$ and Haishuang Zhao ${ }^{1}$ \\ 1. Institute of Inorganic and Analytical Chemistry, Johannes Gutenberg-University, Mainz, Germany
}

Many important materials, ranging from minerals or catalysts to framework compounds and pharmaceuticals are not suitable for growing large crystals prohibiting single crystal X-ray analysis. Yet, introduction of nano crystallinity and special crystallographic features like disorder, defects, pseudo symmetry or stress/strain effects creates new or allows optimizing existing physical properties. With increasing complexity of the structures and special structural features as well as with decreasing size of crystalline domains, X-ray powder diffraction becomes more and more difficult for structural characterization, which is fundamental for understanding material properties. High-resolution transmission electron microscopy (HR-(S)TEM) allows visualizing structural features directly at the atomic scale but requires high electron dose of several thousand $\mathrm{e}^{-} / \AA^{2} \mathrm{~s}$ causing beam damage. In contrast, electron diffraction needs only a fraction of this electron dose. For a complete structure solution, delivering atomic positions in sub Ångstrom accuracy, needs three-dimensional experimental data with high completeness. Data collection from oriented nano crystals limits the amount of measurable reflections significant and thus, delivers mostly heavy atom positions but hardly lighter atoms. Dynamical scattering effects strongly enhanced in oriented zones and may be reduced by electron beam precession technique [1]

Automated electron diffraction tomography [2] uses diffraction patterns from non-oriented nano crystals taken during a fixed tilt sequence. In such a way electron diffraction data collection of most of the independent reflections is possible with significantly reduced dynamical effects. Additionally, in order to integrate reflections fully the electron beam is precessed. The resulting diffraction patterns cannot be analyzed manually any more, thus a dedicated software was programmed (ADT3D / eADT [3]) which, after some geometrical corrections, reconstructs the three-dimensional reciprocal space. From the 3D volume, cell parameters can be determined using clustering-routines. Subsequently, reflections indexing and intensities integration is performed. The three-dimensional reciprocal space reconstruction provides additionally the chance to inspect the volume by eye and detect crystallographic specialties such as disorder, twinning or additional individuals.

Experimental settings: ADT data set collection with a FISCHIONE tomography holder and a cooled GATAN single-tilt holder on a Tecnai F30 S-TWIN transmission electron microscope equipped with a field emission gun working at $300 \mathrm{kV}$. For crystal tracking STEM images collected by a FISCHIONE high angular annular dark field detector (HAADF) were used allowing to stay continuously in diffraction mode. Nano electron diffraction patterns were acquired with a CCD camera (14-bit GATAN 794MSC). A mild illumination setting provides an electron dose rate of $10-15 \mathrm{e} / \AA^{2} \mathrm{~s}$. Nano electron diffraction was performed employing a $10 \mu \mathrm{m}$ C2 condenser aperture with a 100-50 nm beam on the sample. Further improvement in reflection intensity quality is possible coupling ADT with precession electron diffraction (DigiStar unit, NanoMEGAS). The precession angle kept at $1.2^{\circ}$ is significantly smaller than for precessed in-zone diffraction patterns [4].

ADT data sets are of higher quality than those collected by conventional electron diffraction based on 
oriented patterns. Dynamical effects are reduced to such extend that a standard kinematic approach (intensities proportional to $\mathrm{Fhkl}^{2}$ ) delivers ab-initio the complete structural model solved by direct methods or simulated annealing. Based on ADT and ADT/PED data it was possible to solve a wide range of crystal structures from nano particles down to 30nm [5]. Remarkably, large cell porous minerals, zeolites (doped and calcinated), highly beam-sensitive metal-organic frameworks, organicinorganic hybrids, small organic molecules as well as quasicrystal approximants [6] have been successfully solved. Moreover, in the case of human tooth hydroxyapatite ADT analysis resulted in noncentrosymmetric structures later confirmed using convergent beam electron diffraction [7].

For mineralogical quests, ADT turned out to be extremely helpful for detecting new minerals existing only in very small amounts [8]. Recent structure solutions cover the investigation of samples synthesized under high pressure in multi anvil cells. For samples prepared by ion-milling as FIB lamellae the visualization in STEM mode and the use of nano beam diffraction is extremely helpful enabling data collection only from one grain [9]. The crystal structure of a novel boron oxynitride (BON) was solved based on ADT data allowing even for a direct refinement of the twinned structure [10].

References:

[1] Vincent, R., Midgley, P.A., Ultramicroscopy, 53 (1994), p. 271

[2] Kolb, U. et al, Ultramicroscopy, 107 (2007), p. 507

[3] Kolb, U., Gorelik, T., Otten, M.T., Ultramicroscopy, 108 (2008), p. 763

[4] Mugnaioli, E., Gorelik, T., Kolb, U., Ultramicroscopy, 109 (2009), p. 758

[5] Mugnaioli E. et al, Angewandte Chem.Int. Ed., 51(28) (2012), p. 7041

[6] S. Samuha et al, Acta Cryst. B 70(6) (2014), p. 999

[7] E. Mugnaioli et al, Chem. Eur. J. 20 (2014), p. 6849

[8] G. C. Capitani et al, American Mineralogist, 99(2-3) (2014), p. 500

[9] S. Bhat et al, Chem. Mater. 27 (2015), p. 5907

[10] M. Koch-Müller et al, American Mineralogist, 99(11-12) (2014), p. 2405 